\title{
The Comparison of Seasonal Ginsenoside Composition Contents in Korean Wild Simulated Ginseng (Panax ginseng) which were Cultivated in Different Areas and Various Ages
}

\author{
Byung Wook Yang ${ }^{1, \dagger}$, Jae Bum Lee ${ }^{2, \uparrow}$, Jung Min Lee ${ }^{2}$, Min Su Jo², Jae Kyung Byun ${ }^{3}$, \\ Hyoung Chun $\mathrm{Kim}^{4, *}$, and Sung Kwon $\mathrm{Ko}^{2, *}$ \\ ${ }^{1}$ Leaders in INdustry-university Cooperation + (LINC+), Semyung University, Jecheon 27136, Korea \\ ${ }^{2}$ The Department of Oriental Medical Food \& Nutrition, Semyung University, Jecheon 27136, Korea \\ ${ }^{3}$ Korea Forestry Promotion Institute, Seoul 07570, Korea \\ ${ }^{4}$ College of Pharmacy, Kangwon National University, Chunchon 24341, Korea
}

\begin{abstract}
The ginsenoside content was compared with wild simulated ginseng (Panax ginseng) collected every season at 11 wild simulated ginseng plantations in Korea. As a result, the total saponin of 7 years old wild simulated ginseng showed the highest content of $4.5 \%$ in spring sampling wild simulated ginseng, $2.0 \%$ in summer sampling wild simulated ginseng, $1.2 \%$ in winter sampling wild simulated ginseng and $1.0 \%$ in autumn sampling wild simulated ginseng. And also, the total saponin of 10 years old wild simulated ginseng showed the highest content of $3.9 \%$ in spring sampling wild simulated ginseng, summer sampling wild simulated ginseng $(1.8 \%)$, winter sampling wild simulated ginseng $(1.6 \%)$ and autumn sampling wild simulated ginseng $(0.6 \%)$. Therefore, the total saponin of spring sampling wild simulated ginseng was about $4.5-6.5$ times higher than that of autumn sampling wild simulated ginseng regardless of cultivation period.
\end{abstract}

Keywords - Ginsenoside, Wild simulated ginseng, Panax ginseng, Season, Spring sampling, Autumn sampling

\section{Introduction}

Ginseng (Panax ginseng C. A. Meyer) is a edible herb medicine in oriental medicine that has been passed down as a top medicine in the oldest medical herb book in orient of Sin-nong-bon-cho-gyeong (B.C. 3300) and also, wild ginseng reseeded in mountains throughout history was collected and used as a edible herb medicine by the name of ginseng. According to the need for cultivation as a results of exhaustion due to over-catching, the wild simulated ginseng came to be cultivated and industrialized to the ginseng cultivated in fields and paddies.

According to Do-gyeong-bon-cho (A.D. 1061) compiled at the time of Injong of Song dynasty in China as a

\footnotetext{
*Author for correspondence

Sung Kwon Ko, Department of Oriental Medical Food \& Nutrition, Semyung University, Jecheon 27136, Korea

Tel: +82-43-649-1433; E-mail: skko@semyung.ac.kr

Hyoung Chun Kim, College of Pharmacy, Kangwon National University, Chunchon 24341, Korea

Tel: +82-33-250-6917; E-mail: kimhc@kangwon.ac.kr

${ }^{\dagger}$ Byung Wook Yang and Jae Bum Lee were equal contributors to this manuscript; order of listing was determined alphabetically.
}

bibliographic study on the cultivation history of wild simulated ginseng and ginseng, cultivation of wild simulated ginseng is considered to have started then through the record of external features according to the growth years for 4 year-, 5 year- and 10 year-old Korean ginseng. As the wild ginseng and the ginseng were recorded together in 'Jiriji' of Sejong Jangheon Daewang Annals of Sejong era (A.D. 1419-1450) of Chosun Dynasty, it appears to be attributable to the recognition for the need to distinguish wild ginseng from cultivated ginseng.

In addition, while undergoing Sukjong era (A.D. 16751720), the cultivation technology began to be shared through compilation, distribution, etc. of books concerning ginseng cultivation techniques, and prevalence of ginseng cultivation can be affirmed through recording of terms such as ginseng field, etc. Jeongjo Annal of Jeongjo era (A.D. 1777-1800) records that house ginseng (cultivated ginseng) was openly cultivated near the houses, being expanded nationwide.

Furthermore, Ko et al. ${ }^{1,2}$ reported into oriental medicine literature that the time for the sign drug nature of ginseng to be changed from mild cool to warm had A.D. 1600 as a starting point, which suggests that the change in 
cultivation environments for wild simulated ginseng and cultivated ginseng was exhibited as the change in the nature of the drug. ${ }^{1,2}$ Currently, the ginseng reseeded in mountains is called mountain cultivated ginseng, the ginseng cultivated in fields and paddies called cultivated ginseng, and the ginseng where seeds of ginseng and mountain ginseng are sown in mountains for cultivation under natural light, no pesticide and no fertilizing called wild simulated ginseng.

Major physiology activation substances for the wild simulated ginseng and the ginseng are known to contain ginsenosides, polyacetylenes, ginseng proteins, polysaccharides, and phenolic compounds. ${ }^{3-6}$ The ginsenoside is noted as an effective composition of ginseng exhibiting biochemical and pharmacological actions so that many studies focused on this are being conducted, and its chemical structures have been clearly verified by studies of Shibata et al. ${ }^{78}$ since the full-pledged start in the latter 1960's. Also, according to the characteristics of chemical structure, classification is being made into protopanaxadiol (PD)-based, protopanaxatriol (PT)-based, and oleanane-based saponin.

Meanwhile, the wild simulated ginseng is known to have higher effects in the efficacy aspect than the cultivated ginseng, and the wild simulated ginseng is evaluated to have high efficacy below the natural mountain ginseng in herb medicine prescriptions. When compared with the cultivated ginseng, the wild simulated ginseng has been reported to have a higher content of ginsenoside as the ginseng saponin, and is known to be more outstanding in the efficacy aspect than the ginseng cultivated in fields and paddies. ${ }^{9,10}$

Ginsenoside contents are lower in the case of concentrated cultivation in cultivation lands, and the wild simulated ginseng grown wild in China and Korea has been reported to have more outstanding efficacy such as cancer prevention, etc. ${ }^{11}$ The wild simulated ginseng is being discovered to have efficacy in anticancer, ${ }^{12}$ drop in blood pressure, ${ }^{13}$ anti-oxidation, ${ }^{14}$ improvement in liver function, ${ }^{15}$ lipid drop, ${ }^{16}$ etc., and has its efficacy being acknowledged to be next to the natural mountain ginseng in pharmacological activity aspect.

Recently, composition studies for ginseng per year and region ${ }^{17,18}$ as well as composition studies for distinction between the wild simulated ginseng and the cultivated ginseng ${ }^{19,20}$ are also being conducted in part. However, based on the fact that studies on the wild simulated ginseng per season are scarce, identity of our wild simulated ginseng will be verified in the present study by performing a comparison and analysis study concerning ginsenosides of the wild simulated ginseng produced in Korea per season.

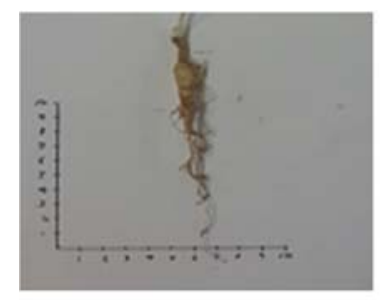

A

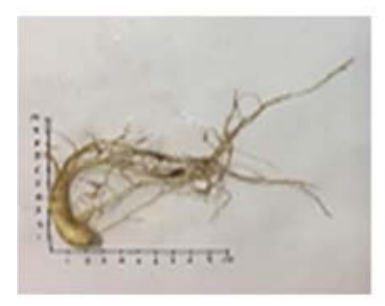

E

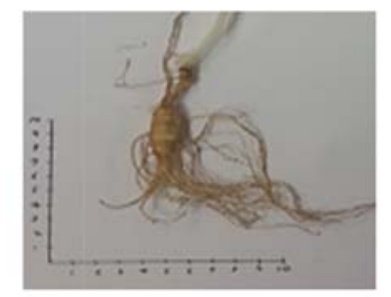

B

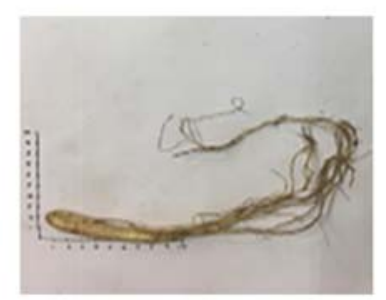

F

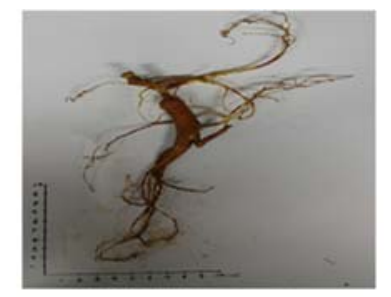

C

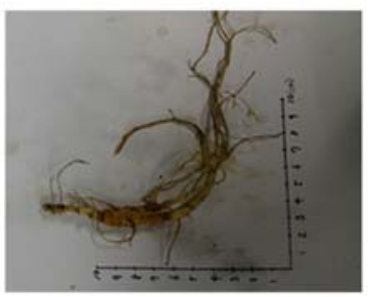

G

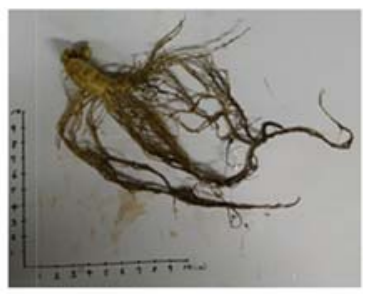

D

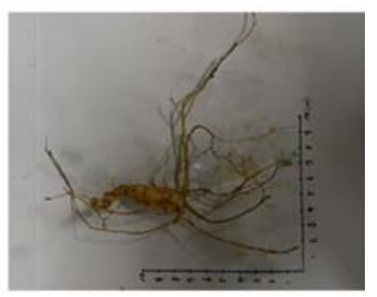

H

Fig. 1. Photographs of the A : 7 years old wild simulated ginseng collected spring in Dan-Yang, B : 10 years old wild simulated ginseng collected spring in Dan-Yang, C : 7 years old wild simulated ginseng collected summer in Geo-Chang, D : 10 years old wild simulated ginseng collected summer in Geo-Chang, E : 7 years old wild simulated ginseng collected autumn in Mu-Ju, F : 10 years old wild simulated ginseng collected autumn in Mu-Ju, G : 7 years old wild simulated ginseng collected winter in Gong-Ju, H : 10 years old wild simulated ginseng collected winter in Gong-Ju used in the study. 


\section{Experimental}

Materials - As shown in Fig. 1, the 7 years old and 10 years old wild simulated ginsengs grown at several areas in South Korea. Pyeongchang (PC) in Gangwon and Namyangju (NYJ) in Gyeonggi province are the northcentral region of South Korea. Danyang (DY) in Chungbuk, and Gongju (GJ), Geumsan (GS) and Seosan (SS) in Chungnam province are the middle region of South Korea. Moreover, Namwon (NW), Muju (MJ) and Imsil (IS) in Jeonbuk, Yeongju (YJ) in Gyeongbuk, and Geochang (GC) in Gyeongnam province are the southern region of South Korea. The specimens were collected in each spring (May), summer (July), autumn (September) and winter (November), and then stored at the oriental medical food research laboratory of Semyung University.

Preparation of Extract - Each wild simulated ginseng is freeze-dried and its rhizome is removed. The ginseng is ground to powder, then $70 \%$ ethyl alcohol $200 \mathrm{ml}$ is added to each $3 \mathrm{~g}$ of the ginseng powder and reflux extraction is performed by repeating 2-hour course 4 times. Filtering and conducting vacuum evaporation. Finally, acquiring the $70 \%$ ethyl alcohol extract.

Preparation of Crude Saponin ${ }^{21}$ - Adding each 70\% ethyl alcohol extract acquired above to ethyl ether $50 \mathrm{ml}$ and conducting the ultrasonic waves extraction by repeating one-hour course 3 times. Doing centrifugation and getting rid of supernatant. Adding bubbled butanol $50 \mathrm{ml}$ to the residue and conducting the ultrasonic waves extraction by repeating two-hour course 3 times. Collecting butanol layers acquired by centrifugation and conducting vacuum evaporation. Finally acquiring crude saponin.

HPLC Analysis of Ginsenoside Content of Wild Simulated Ginseng-Ginsenoside composition of the concentrate was analyzed by HPLC according to the method of Ko et al. ${ }^{22}$ The total ginsenoside content and ginsenoside composition of each sample were analyzed three times. The pure ginsenoside standards ( $99 \%$ purity) used in this experiment were purchased from Chromadex (St. Santa Ana, CA, USA) and Ambo Institute (Seoul, South Korea). The HPLC instrument was Waters 1525 binary HPLC system (Boston, MA, USA), and the column was Eurospher 100-5 C18 (Knauer, $250 \times 3 \mathrm{~mm}$, Germany). Mobile phase was the mixture of acetonitrile and distilled water (J.T. Baker, NJ, and USA). The content of acetonitrile was sequentially increased from $17 \%$ to $25 \%$ (25 $\mathrm{min}$ ), $24 \%$ to $40 \%$ (50 $\mathrm{min}$ ), $40 \%$ to $60 \%$ (105 $\mathrm{min}$ ), $60 \%$ to $100 \%$ (110 $\mathrm{min}), 100 \%$ to $100 \%(120 \mathrm{~min})$ and adjusted from $100 \%$ to $17 \%$ (125 min, stay for $10 \mathrm{~min}$ ) again in the last. Operating temperature was set to room temperature, and the flow rate was $0.8 \mathrm{~mL} / \mathrm{min}$. The elution profile on chromatogram was obtained by using a UV/VIS detector at $203 \mathrm{~nm}$ (Boston, MA, USA).

Statistical Analysis - Data are presented as mean \pm standard deviation with $\mathrm{n}=3$. A one-way ANOVA determined whether the results differed significantly. Duncan's test was used to identify differences. The level of statistical significance was set at $\mathrm{P}<0.05$. The statistical procedures were performed using SPPS ver. 18.0 software (SPSS Inc., Chicago, IL, USA).

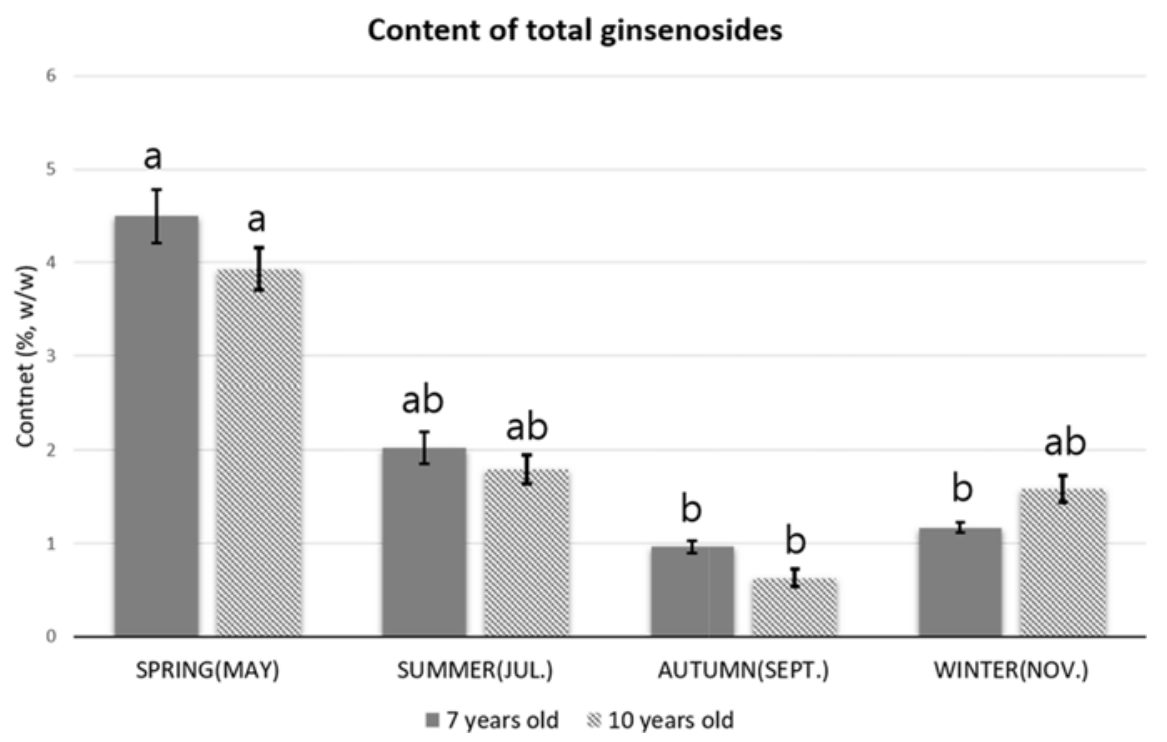

Fig. 2. Seasonal total ginsenoside of Korean wild simulated ginseng at various ages. Results are mean \pm standard deviation. Different letters $(a, b)$ above the bars indicate significant differences between the groups based on the post-hoc Duncan's test results. 


\section{Results and Discussion}

In the present study, basic information of ginsenosides

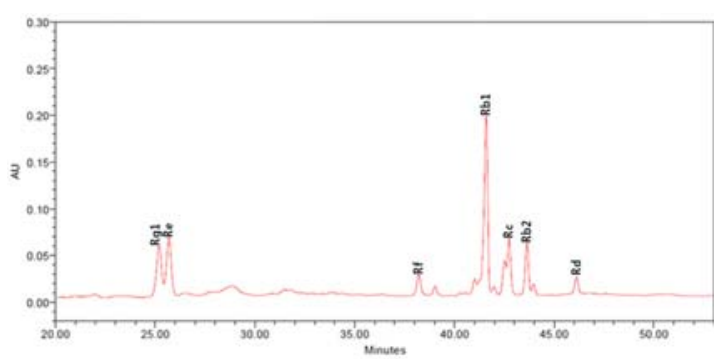

A

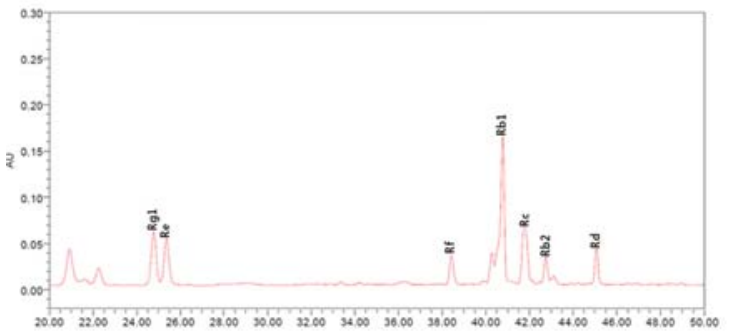

C

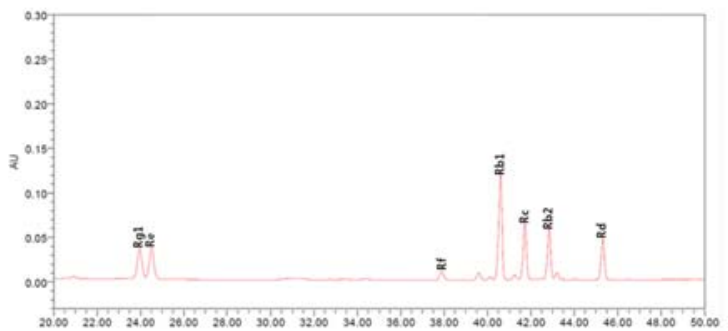

$\mathbf{E}$

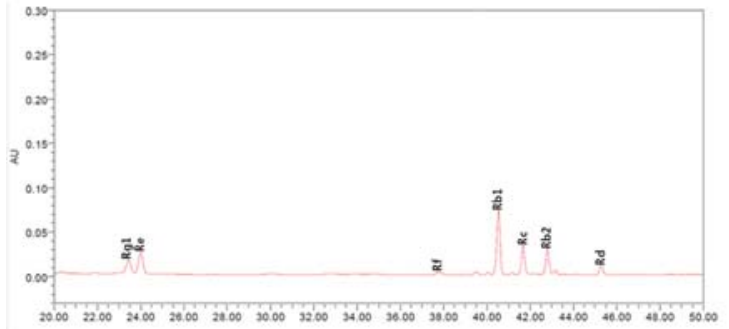

G composition will be provided by reviewing differences with focus on saponin-containing patterns through survey and comparison of individual ginsenoside content distri-

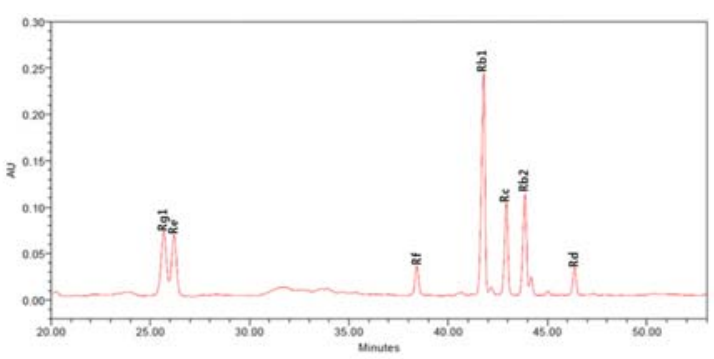

B

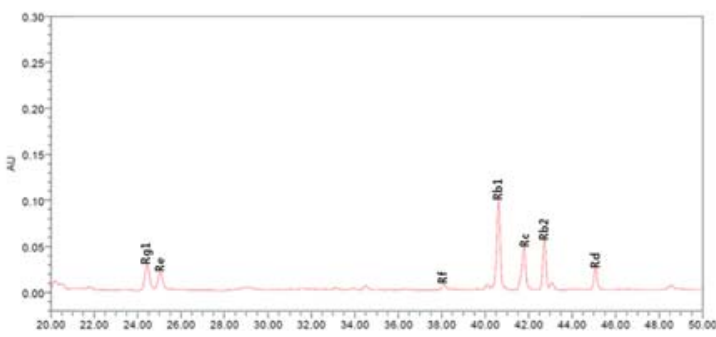

D

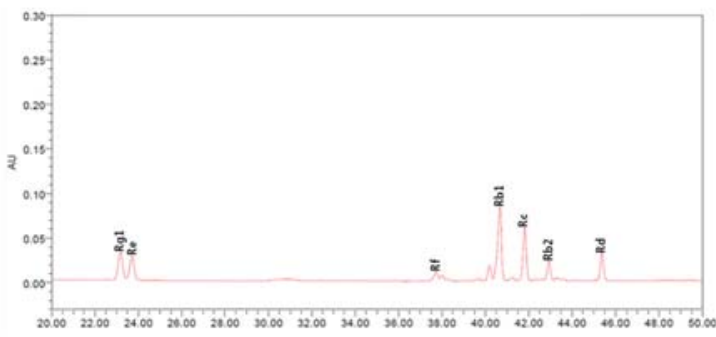

F

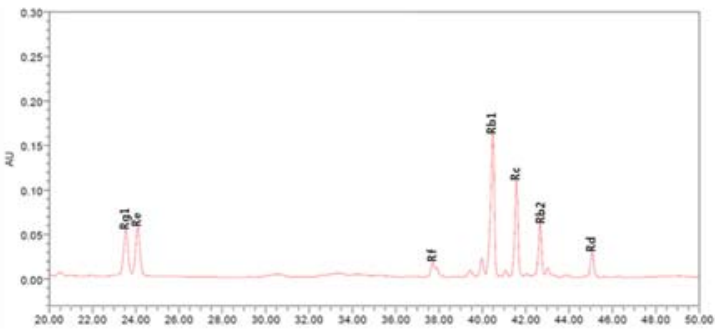

H

Fig. 3. HPLC chromatogram of ginsenosides in the Korean wild simulated ginseng. A : 7 years old wild simulated ginseng collected spring in Dan-Yang, B : 10 years old wild simulated ginseng collected spring in Dan-Yang, C: 7 years old wild simulated ginseng collected summer in Geo-Chang, D : 10 years old wild simulated ginseng collected summer in Geo-Chang, E : 7 years old wild simulated ginseng collected autumn in Mu-Ju, F : 10 years old wild simulated ginseng collected autumn in Mu-Ju, G : 7 years old wild simulated ginseng collected winter in Gong-Ju, H : 10 years old wild simulated ginseng collected winter in Gong-Ju. 
butions for the subject of wild simulated ginseng per season, year and region (11 production places including Geochang, Gongju, Geumsan, Namyangju, Namwon, Danyang, Muju, Seosan, Yeongju, Imsil, and Pyeongchang). The analyzed ginseng saponins were ginsenoside $\mathrm{Rb} 1, \mathrm{Rb} 2, \mathrm{Rc}, \mathrm{Rd}, \mathrm{Re}, \mathrm{Rf}$, and $\mathrm{Rg} 1$, and directly compared with samples through HPLC as shown in Fig. 3, and verified with the average being calculated by statistical processing. ${ }^{19}$

According to the results of comparative analysis of saponin contents per region for the subject of $70 \%$ ethanol extract of the wild simulated ginseng collected in spring (May). Pyeongchang products showed the highest content at $8.6 \%$ followed by Danyang product $(6.1 \%)$ and Muju product $(5.4 \%)$ in the analysis results of ginseng saponin for the subject of 7 years old roots first in total saponin contents as a total sum of individual ginsenosides as shown in Tables 1 and 2. As for 10 years old roots, Namyangju product showed the highest content at $6.9 \%$, followed by Geochang product $(5.0 \%)$ and Danyang product $(5.0 \%)$.

In the total saponin contents of the wild simulated ginseng collected in summer (July), according to the analysis results for ginseng saponin per region for the subject of 7 years old roots s shown in Tables 3 and 4, Danyang product and Gongju product showed the highest

Table 1. The comparison of ginsenoside composition contents in 7 years old Korean wild simulated ginseng (Panax ginseng) collected in spring (May) which were cultivated in different areas

\begin{tabular}{|c|c|c|c|c|c|c|c|c|c|c|c|}
\hline \multirow[b]{2}{*}{ Ginsenosides } & \multicolumn{11}{|c|}{ 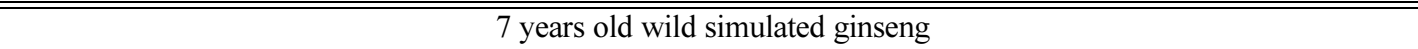 } \\
\hline & $\begin{array}{l}\text { SP7 } \\
\text { GC }\end{array}$ & $\begin{array}{c}\text { SP7 } \\
\text { GJ }\end{array}$ & $\begin{array}{r}\text { SP7 } \\
\text { GS }\end{array}$ & $\begin{array}{l}\text { SP7 } \\
\text { NYJ }\end{array}$ & $\begin{array}{l}\text { SP7 } \\
\text { NW }\end{array}$ & $\begin{array}{l}\text { SP7 } \\
\text { DY }\end{array}$ & $\begin{array}{l}\text { SP7 } \\
\text { MJ }\end{array}$ & $\begin{array}{c}\text { SP7 } \\
\text { SS }\end{array}$ & $\begin{array}{r}\text { SP7 } \\
\text { YJ }\end{array}$ & $\begin{array}{c}\text { SP7 } \\
\text { IS }\end{array}$ & $\begin{array}{r}\text { SP7 } \\
\text { PC }\end{array}$ \\
\hline $\mathrm{Rb}_{1}$ & $1.2 \pm 0.1$ & $2.3 \pm 0.0$ & $1.6 \pm 0.1$ & $2.2 \pm 0.0$ & $1.9 \pm 0.1$ & $2.8 \pm 0.0$ & $2.0 \pm 0.0$ & $1.5 \pm 0.0$ & $1.9 \pm 0.0$ & $0.6 \pm 0.0$ & $3.2 \pm 0.1$ \\
\hline $\mathrm{Rb}_{2}$ & $0.4 \pm 0.0$ & $0.5 \pm 0.0$ & $0.2 \pm 0.0$ & $0.5 \pm 0.0$ & $0.5 \pm 0.0$ & $0.6 \pm 0.0$ & $0.6 \pm 0.0$ & $0.3 \pm 0.0$ & $0.7 \pm 0.0$ & $0.1 \pm 0.0$ & $1.0 \pm 0.0$ \\
\hline $\mathrm{Rc}$ & $0.3 \pm 0.0$ & $0.4 \pm 0.0$ & $0.3 \pm 0.0$ & $0.4 \pm 0.0$ & $0.5 \pm 0.1$ & $0.6 \pm 0.0$ & $0.6 \pm 0.0$ & $0.4 \pm 0.0$ & $0.5 \pm 0.0$ & $0.1 \pm 0.0$ & $0.7 \pm 0.1$ \\
\hline $\mathrm{Rd}$ & $0.1 \pm 0.0$ & $0.1 \pm 0.0$ & $0.2 \pm 0.0$ & $0.2 \pm 0.0$ & $0.2 \pm 0.0$ & $0.2 \pm 0.0$ & $0.3 \pm 0.0$ & $0.1 \pm 0.0$ & $0.2 \pm 0.0$ & $0.1 \pm 0.0$ & $0.8 \pm 0.0$ \\
\hline $\operatorname{Re}$ & $0.3 \pm 0.0$ & $0.5 \pm 0.0$ & $0.7 \pm 0.1$ & $0.6 \pm 0.0$ & $0.5 \pm 0.0$ & $1.0 \pm 0.0$ & $0.8 \pm 0.0$ & $0.7 \pm 0.0$ & $0.4 \pm 0.0$ & $0.7 \pm 0.3$ & $1.4 \pm 0.0$ \\
\hline $\mathrm{Rf}$ & $0.1 \pm 0.0$ & $0.1 \pm 0.0$ & $0.2 \pm 0.0$ & $0.1 \pm 0.0$ & $0.1 \pm 0.0$ & $0.2 \pm 0.0$ & $0.2 \pm 0.0$ & $0.1 \pm 0.0$ & $0.1 \pm 0.0$ & $0.0 \pm 0.0$ & $0.5 \pm 0.0$ \\
\hline $\mathrm{Rg}_{1}$ & $0.3 \pm 0.0$ & $0.6 \pm 0.0$ & $0.4 \pm 0.0$ & $0.6 \pm 0.0$ & $0.5 \pm 0.0$ & $0.8 \pm 0.0$ & $0.8 \pm 0.1$ & $0.5 \pm 0.0$ & $0.5 \pm 0.0$ & $0.2 \pm 0.0$ & $1.0 \pm 0.0$ \\
\hline Total ginsenosides ${ }^{a}$ & 2.6 & 4.5 & 3.7 & 4.7 & 4.1 & 6.1 & 5.4 & 3.6 & 4.3 & 1.9 & 8.6 \\
\hline Diol/ Triol ${ }^{\mathrm{b})}$ & 2.6 & 2.9 & 1.7 & 2.4 & 2.8 & 2.0 & 2.0 & 1.8 & 3.3 & 1.0 & 1.9 \\
\hline
\end{tabular}

* SP7 : Spring collection 7 years old, GC : Geo-Chang, GJ : Gong-Ju, GS : Geum-San, NYJ : Nam-Yang-Ju, NW : Nam-Won, DY : DanYang, MJ : Mu-Ju, SS : Seo-San, YJ : Young-Ju, IS : Im-Sil, PC : Pyeong-Chang, ${ }^{\text {a) }}$ Sum of individual ginsenosides content, ${ }^{\text {b) }}$ Ginsenoside $\mathrm{Rb}_{1}+\mathrm{Rb}_{2}+\mathrm{Rc}+\mathrm{Rd} / \mathrm{Re}+\mathrm{Rf}+\mathrm{Rg}_{1}$, Values represent the mean \pm S.D. $(\mathrm{n}=3)$

Table 2. The comparison of ginsenoside composition contents in 10 years old Korean wild simulated ginseng (Panax ginseng) collected in spring (May) which were cultivated in different areas

$(\%, \mathrm{w} / \mathrm{w})$

\begin{tabular}{|c|c|c|c|c|c|c|c|c|c|c|c|}
\hline \multirow[b]{2}{*}{ Ginsenosides } & \multicolumn{11}{|c|}{10 years old wild simulated ginseng } \\
\hline & $\begin{array}{c}\text { SP10 } \\
\text { GC }\end{array}$ & $\begin{array}{c}\text { SP10 } \\
\text { GJ }\end{array}$ & $\begin{array}{c}\text { SP10 } \\
\text { GS }\end{array}$ & $\begin{array}{l}\text { SP10 } \\
\text { NYJ }\end{array}$ & $\begin{array}{l}\text { SP10 } \\
\text { NW }\end{array}$ & $\begin{array}{c}\text { SP10 } \\
\text { DY }\end{array}$ & $\begin{array}{c}\text { SP10 } \\
\text { MJ }\end{array}$ & $\begin{array}{c}\text { SP10 } \\
\text { SS }\end{array}$ & $\begin{array}{c}\text { SP10 } \\
\text { YJ }\end{array}$ & $\begin{array}{l}\text { SP10 } \\
\text { IS }\end{array}$ & $\begin{array}{c}\text { SP10 } \\
\text { PC }\end{array}$ \\
\hline $\mathrm{Rb}_{1}$ & $2.1 \pm 0.0$ & $2.1 \pm 0.0$ & $1.0 \pm 0.0$ & $3.1 \pm 0.2$ & $2.0 \pm 0.0$ & $2.3 \pm 0.0$ & $1.1 \pm 0.1$ & $1.2 \pm 0.0$ & $1.3 \pm 0.1$ & $0.8 \pm 0.0$ & $1.4 \pm 0.0$ \\
\hline $\mathrm{Rb}_{2}$ & $0.5 \pm 0.0$ & $0.6 \pm 0.0$ & $0.3 \pm 0.0$ & $0.9 \pm 0.1$ & $0.5 \pm 0.0$ & $0.7 \pm 0.0$ & $0.3 \pm 0.0$ & $0.2 \pm 0.0$ & $0.3 \pm 0.0$ & $0.2 \pm 0.0$ & $0.5 \pm 0.0$ \\
\hline $\mathrm{Rc}$ & $0.4 \pm 0.0$ & $0.5 \pm 0.0$ & $0.3 \pm 0.0$ & $0.7 \pm 0.1$ & $0.5 \pm 0.0$ & $0.6 \pm 0.0$ & $0.3 \pm 0.0$ & $0.2 \pm 0.0$ & $0.3 \pm 0.0$ & $0.2 \pm 0.0$ & $0.4 \pm 0.0$ \\
\hline $\mathrm{Rd}$ & $0.4 \pm 0.0$ & $0.1 \pm 0.0$ & $0.1 \pm 0.0$ & $0.3 \pm 0.0$ & $0.2 \pm 0.0$ & $0.2 \pm 0.0$ & $0.2 \pm 0.0$ & $0.2 \pm 0.0$ & $0.2 \pm 0.0$ & $0.1 \pm 0.0$ & $0.2 \pm 0.0$ \\
\hline $\operatorname{Re}$ & $0.8 \pm 0.0$ & $0.5 \pm 0.0$ & $0.5 \pm 0.0$ & $0.7 \pm 0.0$ & $0.7 \pm 0.1$ & $0.5 \pm 0.0$ & $0.4 \pm 0.0$ & $0.4 \pm 0.0$ & $0.4 \pm 0.0$ & $0.5 \pm 0.0$ & $0.5 \pm 0.0$ \\
\hline $\mathrm{Rf}$ & $0.3 \pm 0.0$ & $0.1 \pm 0.0$ & $0.1 \pm 0.0$ & $0.2 \pm 0.0$ & $0.2 \pm 0.0$ & $0.1 \pm 0.0$ & $0.2 \pm 0.0$ & $0.2 \pm 0.0$ & $0.1 \pm 0.0$ & $0.1 \pm 0.0$ & $0.2 \pm 0.0$ \\
\hline $\mathrm{Rg}_{1}$ & $0.5 \pm 0.0$ & $0.5 \pm 0.0$ & $0.3 \pm 0.0$ & $1.1 \pm 0.1$ & $0.5 \pm 0.1$ & $0.6 \pm 0.0$ & $0.4 \pm 0.0$ & $0.4 \pm 0.0$ & $0.3 \pm 0.0$ & $0.3 \pm 0.0$ & $0.6 \pm 0.0$ \\
\hline Total ginsenosides ${ }^{\text {a) }}$ & 5.0 & 4.4 & 2.7 & 6.9 & 4.7 & 5.0 & 2.9 & 2.7 & 3.1 & 2.1 & 3.8 \\
\hline Diol/Triol ${ }^{\mathrm{b})}$ & 2.1 & 3.0 & 2.0 & 2.7 & 2.1 & 3.0 & 2.0 & 1.7 & 2.3 & 1.5 & 2.1 \\
\hline
\end{tabular}

* SP10 : Spring collection 10 years old, GC : Geo-Chang, GJ : Gong-Ju, GS : Geum-San, NYJ : Nam-Yang-Ju, NW : Nam-Won, DY : Dan-Yang, MJ : Mu-Ju, SS : Seo-San, YJ : Young-Ju, IS : Im-Sil, PC : Pyeong-Chang, ${ }^{\text {a) }}$ Sum of individual ginsenosides content, b) Ginsenoside $\mathrm{Rb}_{1}+\mathrm{Rb}_{2}+\mathrm{Rc}+\mathrm{Rd} / \mathrm{Re}+\mathrm{Rf}+\mathrm{Rg}_{1}$, Values represent the mean \pm S.D. $(\mathrm{n}=3)$ 
Table 3. The comparison of ginsenoside composition contents in 7 years old Korean wild simulated ginseng (Panax ginseng) collected in summer (July) which were cultivated in different areas

$(\%, \mathrm{w} / \mathrm{w})$

\begin{tabular}{|c|c|c|c|c|c|c|c|c|c|c|c|}
\hline \multirow[b]{2}{*}{ Ginsenosides } & \multicolumn{11}{|c|}{7 years old wild simulated ginseng } \\
\hline & $\begin{array}{l}\text { S7 } \\
\text { GC }\end{array}$ & $\begin{array}{l}\text { S7 } \\
\text { GJ }\end{array}$ & $\begin{array}{l}\text { S7 } \\
\text { GS }\end{array}$ & $\begin{array}{c}\text { S7 } \\
\text { NYJ }\end{array}$ & $\begin{array}{c}\text { S7 } \\
\text { NW }\end{array}$ & $\begin{array}{l}\text { S7 } \\
\text { DY }\end{array}$ & $\begin{array}{l}\text { S7 } \\
\text { MJ }\end{array}$ & $\begin{array}{l}\text { S7 } \\
\text { SS }\end{array}$ & $\begin{array}{l}\text { S7 } \\
\text { YJ }\end{array}$ & $\begin{array}{l}\text { S7 } \\
\text { IS }\end{array}$ & $\begin{array}{l}\text { S7 } \\
\text { PC }\end{array}$ \\
\hline $\mathrm{Rb}_{1}$ & $0.8 \pm 0.1$ & $1.6 \pm 0.0$ & $1.2 \pm 0.0$ & $0.3 \pm 0.0$ & $1.2 \pm 0.1$ & $1.5 \pm 0.3$ & $0.6 \pm 0.1$ & $0.4 \pm 0.0$ & $0.8 \pm 0.0$ & $0.4 \pm 0.0$ & $0.5 \pm 0.1$ \\
\hline $\mathrm{Rb}_{2}$ & $0.1 \pm 0.0$ & $0.2 \pm 0.0$ & $0.2 \pm 0.0$ & $0.0 \pm 0.0$ & $0.3 \pm 0.0$ & $0.3 \pm 0.1$ & $0.1 \pm 0.0$ & $0.1 \pm 0.0$ & $0.3 \pm 0.0$ & $0.2 \pm 0.0$ & $0.2 \pm 0.0$ \\
\hline $\mathrm{Rc}$ & $0.1 \pm 0.0$ & $0.2 \pm 0.0$ & $0.5 \pm 0.1$ & $0.0 \pm 0.0$ & $0.4 \pm 0.1$ & $0.3 \pm 0.1$ & $0.1 \pm 0.0$ & $0.1 \pm 0.0$ & $0.3 \pm 0.0$ & $0.1 \pm 0.0$ & $0.2 \pm 0.0$ \\
\hline $\mathrm{Rd}$ & $0.1 \pm 0.0$ & $0.2 \pm 0.0$ & $0.1 \pm 0.0$ & $0.0 \pm 0.0$ & $0.2 \pm 0.0$ & $0.3 \pm 0.1$ & $0.1 \pm 0.0$ & $0.1 \pm 0.0$ & $0.2 \pm 0.0$ & $0.1 \pm 0.0$ & $0.1 \pm 0.0$ \\
\hline $\operatorname{Re}$ & $0.2 \pm 0.0$ & $0.5 \pm 0.0$ & $0.4 \pm 0.0$ & $0.1 \pm 0.0$ & $0.3 \pm 0.0$ & $0.4 \pm 0.1$ & $0.2 \pm 0.0$ & $0.1 \pm 0.0$ & $0.2 \pm 0.0$ & $0.2 \pm 0.0$ & $0.2 \pm 0.0$ \\
\hline $\mathrm{Rf}$ & $0.1 \pm 0.0$ & $0.2 \pm 0.0$ & $0.1 \pm 0.1$ & $0.0 \pm 0.0$ & $0.1 \pm 0.0$ & $0.2 \pm 0.0$ & $0.1 \pm 0.0$ & $0.0 \pm 0.0$ & $0.1 \pm 0.0$ & $0.0 \pm 0.0$ & $0.0 \pm 0.0$ \\
\hline $\mathrm{Rg}_{1}$ & $0.3 \pm 0.0$ & $0.6 \pm 0.0$ & $0.4 \pm 0.0$ & $0.1 \pm 0.0$ & $0.4 \pm 0.0$ & $0.7 \pm 0.1$ & $0.2 \pm 0.0$ & $0.1 \pm 0.0$ & $0.2 \pm 0.0$ & $0.1 \pm 0.0$ & $0.1 \pm 0.0$ \\
\hline Total ginsenosides ${ }^{a}$ & 1.7 & 3.6 & 2.8 & 0.7 & 3.0 & 3.6 & 1.5 & 0.9 & 2.0 & 1.2 & 1.3 \\
\hline Diol/Triol ${ }^{\mathrm{b})}$ & 1.7 & 1.7 & 2.2 & 1.7 & 2.7 & 1.7 & 1.7 & 3.4 & 3.3 & 2.3 & 3.1 \\
\hline
\end{tabular}

* S7 : Summer collection 7 years old, GC : Geo-Chang, GJ : Gong-Ju, GS : Geum-San, NYJ : Nam-Yang-Ju, NW : Nam-Won, DY : DanYang, MJ : Mu-Ju, SS : Seo-San, YJ : Young-Ju, IS : Im-Sil, PC : Pyeong-Chang, ${ }^{\text {a) }}$ Sum of individual ginsenosides content, ${ }^{\text {b) }}$ Ginsenoside $\mathrm{Rb}_{1}+\mathrm{Rb}_{2}+\mathrm{Rc}+\mathrm{Rd} / \mathrm{Re}+\mathrm{Rf}+\mathrm{Rg}_{1}$, Values represent the mean \pm S.D. $(\mathrm{n}=3)$

Table 4. The comparison of ginsenoside composition contents in 10 years old Korean wild simulated ginseng (Panax ginseng) collected in summer (July) which were cultivated in different areas

$(\%, \mathrm{w} / \mathrm{w})$

\begin{tabular}{|c|c|c|c|c|c|c|c|c|c|c|c|}
\hline \multirow{3}{*}{ Ginsenosides } & \multicolumn{11}{|c|}{10 years old wild simulated ginseng } \\
\hline & S10 & S10 & S10 & S10 & $\mathrm{S} 10$ & S10 & S10 & S10 & S10 & S10 & S10 \\
\hline & GC & GJ & GS & NYJ & NW & DY & MJ & SS & YJ & IS & $\mathrm{PC}$ \\
\hline $\mathrm{Rb}_{1}$ & $0.2 \pm 0.0$ & $1.0 \pm 0.0$ & $0.7 \pm 0.0$ & $0.6 \pm 0.0$ & $1.7 \pm 0.0$ & $0.7 \pm 0.0$ & $0.5 \pm 0.0$ & $0.5 \pm 0.0$ & $0.4 \pm 0.0$ & $1.5 \pm 0.0$ & $0.6 \pm 0.0$ \\
\hline $\mathrm{Rb}_{2}$ & $0.1 \pm 0.0$ & $0.3 \pm 0.0$ & $0.2 \pm 0.0$ & $0.1 \pm 0.0$ & $0.6 \pm 0.0$ & $0.3 \pm 0.0$ & $0.2 \pm 0.0$ & $0.1 \pm 0.0$ & $0.1 \pm 0.0$ & $0.4 \pm 0.0$ & $0.3 \pm 0.0$ \\
\hline $\mathrm{Rc}$ & $0.1 \pm 0.0$ & $0.2 \pm 0.0$ & $0.2 \pm 0.0$ & $0.2 \pm 0.0$ & $.0 .4 \pm 0.0$ & $0.2 \pm 0.0$ & $0.1 \pm 0.0$ & $0.2 \pm 0.0$ & $0.2 \pm 0.0$ & $0.3 \pm 0.0$ & $0.2 \pm 0.0$ \\
\hline $\mathrm{Rd}$ & $0.0 \pm 0.0$ & $0.1 \pm 0.0$ & $0.1 \pm 0.0$ & $0.0 \pm 0.0$ & $0.2 \pm 0.0$ & $0.1 \pm 0.0$ & $0.1 \pm 0.0$ & $0.1 \pm 0.0$ & $0.1 \pm 0.0$ & $0.2 \pm 0.0$ & $0.2 \pm 0.0$ \\
\hline $\operatorname{Re}$ & $0.0 \pm 0.0$ & $0.2 \pm 0.0$ & $0.1 \pm 0.0$ & $0.2 \pm 0.0$ & $0.4 \pm 0.0$ & $0.3 \pm 0.0$ & $0.1 \pm 0.0$ & $0.2 \pm 0.0$ & $0.2 \pm 0.0$ & $0.6 \pm 0.0$ & $0.2 \pm 0.0$ \\
\hline $\mathrm{Rf}$ & $0.0 \pm 0.0$ & $0.1 \pm 0.0$ & $0.0 \pm 0.0$ & $0.0 \pm 0.0$ & $0.1 \pm 0.0$ & $0.0 \pm 0.0$ & $0.0 \pm 0.0$ & $0.0 \pm 0.0$ & $0.0 \pm 0.0$ & $0.1 \pm 0.0$ & $0.0 \pm 0.0$ \\
\hline $\mathrm{Rg}_{1}$ & $0.0 \pm 0.0$ & $0.3 \pm 0.0$ & $0.3 \pm 0.0$ & $0.2 \pm 0.0$ & $0.4 \pm 0.0$ & $0.2 \pm 0.0$ & $0.1 \pm 0.0$ & $0.1 \pm 0.0$ & $0.1 \pm 0.0$ & $0.4 \pm 0.0$ & $0.3 \pm 0.0$ \\
\hline Total ginsenosides ${ }^{\mathrm{a})}$ & 0.4 & 2.1 & 1.7 & 1.4 & 3.8 & 1.8 & 1.1 & 1.2 & 1.1 & 3.4 & 1.8 \\
\hline Diol/Triol ${ }^{\text {b) }}$ & 3.6 & 2.6 & 2.6 & 2.1 & 3.7 & 2.7 & 3.6 & 2.6 & 2.3 & 2.4 & 2.7 \\
\hline
\end{tabular}

* S10 : Summer collection 10 years old, GC : Geo-Chang, GJ : Gong-Ju, GS : Geum-San, NYJ : Nam-Yang-Ju, NW : Nam-Won, DY : Dan-Yang, MJ : Mu-Ju, SS : Seo-San, YJ : Young-Ju, IS : Im-Sil, PC : Pyeong-Chang, ${ }^{\text {a) }}$ Sum of individual ginsenosides content, ${ }^{\text {b) }}$ Ginsenoside $\mathrm{Rb}_{1}+\mathrm{Rb}_{2}+\mathrm{Rc}+\mathrm{Rd} / \mathrm{Re}+\mathrm{Rf}+\mathrm{Rg}_{1}$, Values represent the mean \pm S.D. $(\mathrm{n}=3)$

content at $3.6 \%$, followed by Namwon product $(3.0 \%)$ and Geumsan product $(2.8 \%)$. As for 10 years old roots, Namwon product showed the highest content at $3.8 \%$, followed by Imsil product (3.4\%) and Gongju product (2.1\%).

In the total saponin contents of the wild simulated ginseng collected in autumn (September) according to the analysis results for ginseng saponin per region for the subject of 7 years old roots as shown in Tables $5 \& 6$, Gongju product showed the highest content at $1.7 \%$, followed by Geumsan product $(1.5 \%)$ and Geochang product $(1.2 \%)$. In terms of 10 years old roots, Gongju product showed the highest content at $1.9 \%$, followed by Geumsan product (1.8\%) and Pyeongchang product $(0.8 \%)$.
In the total saponin contents of the wild simulated ginseng collected in winter (November) according to the analysis results for ginseng saponin per region for the subject of 7 years old roots as shown in Tables $7 \& 8$, Seosan product showed the highest content at $1.7 \%$, followed by Geochang product (1.5\%) and Namwon product (1.4\%). As for 10 years old roots, Gongju product showed the highest content at $3.5 \%$, followed by Namwon product $(2.9 \%)$ and Geumsan product $(2.6 \%)$.

In addition, in the total saponin contents as the total sum of individual ginsenoside as shown in Tables $9 \& 10$ and Fig. 2. According to the comparative analysis results for average contents of ginseng saponin per season for the 
Table 5. The comparison of ginsenoside composition contents in 7 years old Korean wild simulated ginseng (Panax ginseng) collected in autumn (September) which were cultivated in different areas

$(\%, \mathrm{w} / \mathrm{w})$

\begin{tabular}{|c|c|c|c|c|c|c|c|c|c|c|c|}
\hline \multirow{3}{*}{ Ginsenosides } & \multicolumn{11}{|c|}{7 years old wild simulated ginseng } \\
\hline & A7 & A7 & A7 & A7 & A7 & A7 & A7 & A7 & A7 & A7 & $\begin{array}{l}\text { A7 } \\
\text { PC }\end{array}$ \\
\hline & GC & GJ & GS & NYJ & NW & DY & MJ & SS & YJ & IS & PC \\
\hline $\mathrm{Rb}_{1}$ & $0.5 \pm 0.1$ & $0.8 \pm 0.0$ & $0.6 \pm 0.0$ & $0.3 \pm 0.0$ & $0.4 \pm 0.0$ & $0.5 \pm 0.2$ & $0.4 \pm 0.1$ & $0.2 \pm 0.0$ & $0.3 \pm 0.1$ & $0.1 \pm 0.0$ & $0.3 \pm 0.1$ \\
\hline $\mathrm{Rb}_{2}$ & $0.1 \pm 0.0$ & $0.1 \pm 0.0$ & $0.2 \pm 0.0$ & $0.1 \pm 0.0$ & $0.1 \pm 0.0$ & $0.1 \pm 0.0$ & $0.1 \pm 0.0$ & $0.0 \pm 0.0$ & $0.0 \pm 0.0$ & $0.0 \pm 0.0$ & $0.1 \pm 0.0$ \\
\hline $\mathrm{Rc}$ & $0.2 \pm 0.1$ & $0.2 \pm 0.1$ & $0.2 \pm 0.0$ & $0.1 \pm 0.0$ & $0.1 \pm 0.0$ & $0.2 \pm 0.1$ & $0.1 \pm 0.0$ & $0.1 \pm 0.0$ & $0.1 \pm 0.1$ & $0.1 \pm 0.0$ & $0.1 \pm 0.1$ \\
\hline $\mathrm{Rd}$ & $0.1 \pm 0.0$ & $0.1 \pm 0.0$ & $0.1 \pm 0.0$ & $0.0 \pm 0.0$ & $0.1 \pm 0.0$ & $0.1 \pm 0.0$ & $0.1 \pm 0.0$ & $0.0 \pm 0.0$ & $0.0 \pm 0.0$ & $0.0 \pm 0.0$ & $0.1 \pm 0.0$ \\
\hline $\operatorname{Re}$ & $0.2 \pm 0.1$ & $0.3 \pm 0.1$ & $0.2 \pm 0.1$ & $0.1 \pm 0.0$ & $0.2 \pm 0.1$ & $0.2 \pm 0.0$ & $0.1 \pm 0.0$ & $0.1 \pm 0.0$ & $0.1 \pm 0.0$ & $0.1 \pm 0.0$ & $0.1 \pm 0.0$ \\
\hline $\mathrm{Rf}$ & $0.1 \pm 0.0$ & $0.0 \pm 0.0$ & $0.1 \pm 0.0$ & $0.0 \pm 0.0$ & $0.0 \pm 0.0$ & $0.0 \pm 0.0$ & $0.0 \pm 0.0$ & $0.0 \pm 0.0$ & $0.0 \pm 0.0$ & $0.0 \pm 0.0$ & $0.0 \pm 0.0$ \\
\hline $\mathrm{Rg}_{1}$ & $0.2 \pm 0.0$ & $0.2 \pm 0.1$ & $0.2 \pm 0.1$ & $0.1 \pm 0.0$ & $0.2 \pm 0.1$ & $0.1 \pm 0.0$ & $0.1 \pm 0.0$ & $0.0 \pm 0.0$ & $0.1 \pm 0.0$ & $0.0 \pm 0.0$ & $0.1 \pm 0.0$ \\
\hline Total ginsenosides ${ }^{a}$ & 1.2 & 1.7 & 1.5 & 0.6 & 1.1 & 1.1 & 1.1 & 0.4 & 0.7 & 0.3 & 0.9 \\
\hline Diol/Triol ${ }^{\mathrm{b})}$ & 2.2 & 2.3 & 2.4 & 2.3 & 1.9 & 2.0 & 2.8 & 2.0 & 2.1 & 2.2 & 2.0 \\
\hline
\end{tabular}

* A7 : Autumn collection 7 years old, GC : Geo-Chang, GJ : Gong-Ju, GS : Geum-San, NYJ : Nam-Yang-Ju, NW : Nam-Won, DY : Dan-Yang, MJ : Mu-Ju, SS : Seo-San, YJ : Young-Ju, IS : Im-Sil, PC : Pyeong-Chang, ${ }^{\text {a) }}$ Sum of individual ginsenosides content, b) Ginsenoside $\mathrm{Rb}_{1}+\mathrm{Rb}_{2}+\mathrm{Rc}+\mathrm{Rd} / \mathrm{Re}+\mathrm{Rf}+\mathrm{Rg}_{1}$, Values represent the mean \pm S.D. $(\mathrm{n}=3)$

Table 6. The comparison of ginsenoside composition contents in 10 years old Korean wild simulated ginseng (Panax ginseng) collected in autumn (September) which were cultivated in different areas

$(\%, \mathrm{w} / \mathrm{w})$

\begin{tabular}{|c|c|c|c|c|c|c|c|c|c|c|c|}
\hline \multirow{3}{*}{ Ginsenosides } & \multicolumn{11}{|c|}{10 years old wild simulated ginseng } \\
\hline & A10 & A10 & A10 & A10 & A10 & A10 & A10 & A10 & A10 & A10 & A10 \\
\hline & GC & GJ & GS & NYJ & NW & DY & MJ & SS & YJ & IS & $\mathrm{PC}$ \\
\hline $\mathrm{Rb}_{1}$ & $0.1 \pm 0.0$ & $0.8 \pm 0.1$ & $0.8 \pm 0.1$ & $0.1 \pm 0.0$ & $0.1 \pm 0.0$ & $0.0 \pm 0.0$ & $0.1 \pm 0.0$ & $0.3 \pm 0.0$ & $0.2 \pm 0.0$ & $0.2 \pm 0.0$ & $0.3 \pm 0.0$ \\
\hline $\mathrm{Rb}_{2}$ & $0.0 \pm 0.0$ & $0.2 \pm 0.0$ & $0.2 \pm 0.0$ & $0.0 \pm 0.0$ & $0.0 \pm 0.0$ & $0.0 \pm 0.0$ & $0.0 \pm 0.0$ & $0.0 \pm 0.0$ & $0.0 \pm 0.0$ & $0.0 \pm 0.0$ & $0.1 \pm 0.0$ \\
\hline $\mathrm{Rc}$ & $0.0 \pm 0.0$ & $0.2 \pm 0.0$ & $0.2 \pm 0.0$ & $0.0 \pm 0.0$ & $0.0 \pm 0.0$ & $0.0 \pm 0.0$ & $0.0 \pm 0.0$ & $0.1 \pm 0.0$ & $0.1 \pm 0.0$ & $0.1 \pm 0.0$ & $0.1 \pm 0.0$ \\
\hline $\mathrm{Rd}$ & $0.0 \pm 0.0$ & $0.0 \pm 0.0$ & $0.0 \pm 0.0$ & $0.0 \pm 0.0$ & $0.0 \pm 0.0$ & $0.0 \pm 0.0$ & $0.0 \pm 0.0$ & $0.0 \pm 0.0$ & $0.0 \pm 0.0$ & $0.0 \pm 0.0$ & $0.0 \pm 0.0$ \\
\hline $\operatorname{Re}$ & $0.0 \pm 0.0$ & $0.2 \pm 0.1$ & $0.2 \pm 0.1$ & $0.0 \pm 0.0$ & $0.0 \pm 0.0$ & $0.0 \pm 0.0$ & $0.0 \pm 0.0$ & $0.1 \pm 0.0$ & $0.1 \pm 0.0$ & $0.1 \pm 0.0$ & $0.1 \pm 0.0$ \\
\hline $\mathrm{Rf}$ & $0.0 \pm 0.0$ & $0.1 \pm 0.0$ & $0.1 \pm 0.0$ & $0.0 \pm 0.0$ & $0.0 \pm 0.0$ & $0.0 \pm 0.0$ & $0.0 \pm 0.0$ & $0.0 \pm 0.0$ & $0.0 \pm 0.0$ & $0.0 \pm 0.0$ & $0.0 \pm 0.0$ \\
\hline $\mathrm{Rg}_{1}$ & $0.0 \pm 0.0$ & $0.3 \pm 0.1$ & $0.3 \pm 0.1$ & $0.0 \pm 0.0$ & $0.0 \pm 0.0$ & $0.0 \pm 0.0$ & $0.0 \pm 0.0$ & $0.1 \pm 0.0$ & $0.1 \pm 0.0$ & $0.1 \pm 0.0$ & $0.1 \pm 0.0$ \\
\hline Total ginsenosides $^{\text {a) }}$ & 0.2 & 1.9 & 1.8 & 0.1 & 0.2 & 0.1 & 0.3 & 0.6 & 0.6 & 0.5 & 0.8 \\
\hline Diol/Triol ${ }^{\mathrm{b})}$ & 2.4 & 2.0 & 2.2 & 2.7 & 1.9 & 2.3 & 2.1 & 1.9 & 1.9 & 2.6 & 2.5 \\
\hline
\end{tabular}

* A10 : Autumn collection 10 years old, GC : Geo-Chang, GJ : Gong-Ju, GS : Geum-San, NYJ : Nam-Yang-Ju, NW : Nam-Won, DY : Dan-Yang, MJ : Mu-Ju, SS : Seo-San, YJ : Young-Ju, IS : Im-Sil, PC : Pyeong-Chang, ${ }^{\text {a) }}$ Sum of individual ginsenosides content, ${ }^{\text {b) }}$ Ginsenoside $\mathrm{Rb}_{1}+\mathrm{Rb}_{2}+\mathrm{Rc}+\mathrm{Rd} / \mathrm{Re}+\mathrm{Rf}+\mathrm{Rg}_{1}$, Values represent the mean $\pm \mathrm{S} . \mathrm{D}$. $(\mathrm{n}=3)$

subject of $70 \%$ ethanol extract of the wild simulated ginseng collected from 11 places of South Korea. As a results of the subject of 7 years old roots, the wild simulated ginseng collected in spring showed the highest content at $4.5 \%$ in terms of the analysis results for ginseng saponin per season. It was followed by the wild simulated ginseng collected in summer $(2.0 \%)$, winter $(1.2 \%)$, and autumn (1.0\%). In the ginseng saponin ratio for the protopanaxadiol group and the protopanaxatriol group, while the wild simulated ginseng collected in winter showed a high ratio for the protopanaxadiol group at 3.0, a similar tendency was observed for autumn (2.2), spring (2.2), and summer (2.1). Significance of the difference among the 7 years old groups of each places $(P<0.05)$.

And also, according to the analysis results for ginseng saponin per season for the subject of 10 years old roots, the wild simulated ginseng collected in spring showed the highest content at $3.9 \%$, followed by the wild simulated ginseng collected in summer $(1.8 \%)$, wild simulated ginseng collected in winter $(1.6 \%)$, and wild simulated ginseng collected in autumn $(0.6 \%)$. In the ginseng saponin ratio for protopanaxadiol group and protopanaxatriol group, while the wild simulated ginseng collected in winter showed 2.8 and the wild simulated ginseng collected in summer 2.7 with the content of protopanaxadiol group showing a high ratio, a similar tendency was observed for 
Table 7. The comparison of ginsenoside composition contents in 7 years old Korean wild simulated ginseng (Panax ginseng) collected in winter (November) which were cultivated in different areas

$(\%, \mathrm{w} / \mathrm{w})$

\begin{tabular}{|c|c|c|c|c|c|c|c|c|c|c|c|}
\hline \multirow{3}{*}{ Ginsenosides } & \multicolumn{11}{|c|}{ "7 years old wild simulated ginseng } \\
\hline & W7 & W7 & W7 & W7 & W7 & W7 & W7 & W7 & W7 & W7 & W7 \\
\hline & $\mathrm{GC}$ & GJ & GS & NYJ & NW & DY & MJ & SS & YJ & IS & PC \\
\hline $\mathrm{Rb}_{1}$ & $0.7 \pm 0.0$ & $0.3 \pm 0.0$ & $0.6 \pm 0.0$ & $0.6 \pm 0.0$ & $0.6 \pm 0.0$ & $0.4 \pm 0.0$ & $0.4 \pm 0.0$ & $0.8 \pm 0.0$ & $0.5 \pm 0.0$ & $0.3 \pm 0.0$ & $0.4 \pm 0.0$ \\
\hline $\mathrm{Rb}_{2}$ & $0.2 \pm 0.0$ & $0.1 \pm 0.0$ & $0.2 \pm 0.0$ & $0.1 \pm 0.0$ & $0.1 \pm 0.0$ & $0.1 \pm 0.0$ & $0.2 \pm 0.0$ & $0.1 \pm 0.0$ & $0.0 \pm 0.0$ & $0.1 \pm 0.0$ & $0.2 \pm 0.0$ \\
\hline $\mathrm{Rc}$ & $0.2 \pm 0.0$ & $0.1 \pm 0.0$ & $0.1 \pm 0.0$ & $0.2 \pm 0.0$ & $0.2 \pm 0.0$ & $0.1 \pm 0.0$ & $0.1 \pm 0.0$ & $0.3 \pm 0.0$ & $0.2 \pm 0.0$ & $0.2 \pm 0.0$ & $0.1 \pm 0.0$ \\
\hline $\mathrm{Rd}$ & $0.1 \pm 0.0$ & $0.0 \pm 0.0$ & $0.0 \pm 0.0$ & $0.0 \pm 0.0$ & $0.1 \pm 0.0$ & $0.0 \pm 0.0$ & $0.0 \pm 0.0$ & $0.0 \pm 0.0$ & $0.0 \pm 0.0$ & $0.0 \pm 0.0$ & $0.1 \pm 0.0$ \\
\hline $\operatorname{Re}$ & $0.2 \pm 0.0$ & $0.1 \pm 0.0$ & $0.2 \pm 0.0$ & $0.2 \pm 0.0$ & $0.2 \pm 0.0$ & $0.2 \pm 0.0$ & $0.1 \pm 0.0$ & $0.2 \pm 0.0$ & $0.1 \pm 0.0$ & $0.1 \pm 0.0$ & $0.1 \pm 0.0$ \\
\hline $\mathrm{Rf}$ & $0.0 \pm 0.0$ & $0.0 \pm 0.0$ & $0.0 \pm 0.0$ & $0.0 \pm 0.0$ & $0.0 \pm 0.0$ & $0.0 \pm 0.0$ & $0.0 \pm 0.0$ & $0.0 \pm 0.0$ & $0.0 \pm 0.0$ & $0.0 \pm 0.0$ & $0.0 \pm 0.0$ \\
\hline $\mathrm{Rg}_{1}$ & $0.1 \pm 0.0$ & $0.1 \pm 0.0$ & $0.1 \pm 0.0$ & $0.1 \pm 0.0$ & $0.2 \pm 0.0$ & $0.1 \pm 0.0$ & $0.1 \pm 0.0$ & $0.2 \pm 0.0$ & $0.2 \pm 0.0$ & $0.1 \pm 0.0$ & $0.1 \pm 0.0$ \\
\hline Total ginsenosides ${ }^{a}$ & 1.5 & 0.7 & 1.3 & 1.3 & 1.4 & 1.0 & 0.9 & 1.7 & 1.1 & 0.8 & 1.0 \\
\hline Diol/Triol $^{\text {b) }}$ & 3.4 & 3.3 & 2.8 & 2.9 & 2.5 & 2.3 & 3.9 & 3.0 & 2.9 & 2.4 & 3.7 \\
\hline
\end{tabular}

* W7 : Winter collection 7 years old, GC : Geo-Chang, GJ : Gong-Ju, GS : Geum-San, NYJ : Nam-Yang-Ju, NW : Nam-Won, DY : DanYang, MJ : Mu-Ju, SS : Seo-San, YJ : Young-Ju, IS : Im-Sil, PC : Pyeong-Chang, ${ }^{\text {a) }}$ Sum of individual ginsenosides content, ${ }^{\text {b) }}$ Ginsenoside $\mathrm{Rb}_{1}+\mathrm{Rb}_{2}+\mathrm{Rc}+\mathrm{Rd} / \mathrm{Re}+\mathrm{Rf}+\mathrm{Rg}_{1}$, Values represent the mean \pm S.D. $(\mathrm{n}=3)$

Table 8. The comparison of ginsenoside composition contents in 10 years old Korean wild simulated ginseng (Panax ginseng) collected in winter (November) which were cultivated in different areas

$(\%, \mathrm{w} / \mathrm{w})$

\begin{tabular}{|c|c|c|c|c|c|c|c|c|c|c|c|}
\hline \multirow[b]{2}{*}{ Ginsenosides } & \multicolumn{11}{|c|}{10 years old wild simulated ginseng } \\
\hline & $\begin{array}{c}\text { W10 } \\
\text { GC }\end{array}$ & $\begin{array}{c}\text { W10 } \\
\text { GJ }\end{array}$ & $\begin{array}{l}\text { W10 } \\
\text { GS }\end{array}$ & $\begin{array}{l}\text { W10 } \\
\text { NYJ }\end{array}$ & $\begin{array}{l}\text { W10 } \\
\text { NW }\end{array}$ & $\begin{array}{l}\text { W10 } \\
\text { DY }\end{array}$ & $\begin{array}{c}\text { W10 } \\
\text { MJ }\end{array}$ & $\begin{array}{c}\text { W10 } \\
\text { SS }\end{array}$ & $\begin{array}{c}\text { W10 } \\
\text { YJ }\end{array}$ & $\begin{array}{l}\text { W10 } \\
\text { IS }\end{array}$ & $\begin{array}{l}\text { W10 } \\
\text { PC }\end{array}$ \\
\hline $\mathrm{Rb}_{1}$ & $0.5 \pm 0.0$ & $1.5 \pm 0.0$ & $1.3 \pm 0.0$ & $0.6 \pm 0.0$ & $1.4 \pm 0.0$ & $0.1 \pm 0.0$ & $0.5 \pm 0.0$ & $0.6 \pm 0.0$ & $0.5 \pm 0.0$ & $0.7 \pm 0.0$ & $0.4 \pm 0.0$ \\
\hline $\mathrm{Rb}_{2}$ & $0.0 \pm 0.0$ & $0.4 \pm 0.0$ & $0.2 \pm 0.0$ & $0.0 \pm 0.0$ & $0.2 \pm 0.0$ & $0.0 \pm 0.0$ & $0.1 \pm 0.0$ & $0.1 \pm 0.0$ & $0.1 \pm 0.0$ & $0.1 \pm 0.0$ & $0.1 \pm 0.0$ \\
\hline $\mathrm{Rc}$ & $0.2 \pm 0.0$ & $0.6 \pm 0.0$ & $0.4 \pm 0.0$ & $0.2 \pm 0.0$ & $0.4 \pm 0.0$ & $0.0 \pm 0.0$ & $0.2 \pm 0.0$ & $0.2 \pm 0.0$ & $0.1 \pm 0.0$ & $0.2 \pm 0.0$ & $0.2 \pm 0.0$ \\
\hline $\mathrm{Rd}$ & $0.1 \pm 0.0$ & $0.2 \pm 0.0$ & $0.0 \pm 0.0$ & $0.0 \pm 0.0$ & $0.1 \pm 0.0$ & $0.0 \pm 0.0$ & $0.0 \pm 0.0$ & $0.0 \pm 0.0$ & $0.0 \pm 0.0$ & $0.0 \pm 0.0$ & $0.1 \pm 0.0$ \\
\hline $\operatorname{Re}$ & $0.2 \pm 0.0$ & $0.4 \pm 0.0$ & $0.3 \pm 0.0$ & $0.2 \pm 0.0$ & $0.4 \pm 0.0$ & $0.1 \pm 0.0$ & $0.1 \pm 0.0$ & $0.2 \pm 0.0$ & $0.2 \pm 0.0$ & $0.1 \pm 0.0$ & $0.1 \pm 0.0$ \\
\hline $\mathrm{Rf}$ & $0.0 \pm 0.0$ & $0.1 \pm 0.0$ & $0.1 \pm 0.0$ & $0.0 \pm 0.0$ & $0.1 \pm 0.0$ & $0.0 \pm 0.0$ & $0.0 \pm 0.0$ & $0.0 \pm 0.0$ & $0.0 \pm 0.0$ & $0.0 \pm 0.0$ & $0.0 \pm 0.0$ \\
\hline $\mathrm{Rg}_{1}$ & $0.1 \pm 0.0$ & $0.4 \pm 0.0$ & $0.3 \pm 0.0$ & $0.1 \pm 0.0$ & $0.4 \pm 0.0$ & $0.0 \pm 0.0$ & $0.1 \pm 0.0$ & $0.1 \pm 0.0$ & $0.1 \pm 0.0$ & $0.2 \pm 0.0$ & $0.1 \pm 0.0$ \\
\hline Total ginsenosid & 1.2 & 3.5 & 2.6 & 1.2 & 2.9 & 0.3 & 1.1 & 1.2 & 1.1 & 1.4 & 0.9 \\
\hline Diol/Triol ${ }^{\mathrm{b})}$ & 2.7 & 3.1 & 2.5 & 2.8 & 2.6 & 2.2 & 3.3 & 3.1 & 2.4 & 2.7 & 2.8 \\
\hline
\end{tabular}

* W10 : Winter collection 10 years old, GC : Geo-Chang, GJ : Gong-Ju, GS : Geum-San, NYJ : Nam-Yang-Ju, NW : Nam-Won, DY : Dan-Yang, MJ : Mu-Ju, SS : Seo-San, YJ : Young-Ju, IS : Im-Sil, PC : Pyeong-Chang, ${ }^{\text {a) }}$ Sum of individual ginsenosides content, ${ }^{\text {b) }}$ Ginsenoside $\mathrm{Rb}_{1}+\mathrm{Rb}_{2}+\mathrm{Rc}+\mathrm{Rd} / \mathrm{Re}+\mathrm{Rf}+\mathrm{Rg}_{1}$, Values represent the mean \pm S.D. $(\mathrm{n}=3)$

Table 9. Seasonal ginsenoside average content of 7 years old Korean wild simulated ginseng (11 areas)

$(\%, \mathrm{w} / \mathrm{w})$

\begin{tabular}{ccccc}
\hline \hline Ginsenosides & \multicolumn{4}{c}{ Seasonal 7 years old Korean wild simulated ginseng (11 regions) } \\
\hline $\mathrm{Rb}_{1}$ & spring (May) & summer (July) & autumn (September) & winter (November) \\
$\mathrm{Rb}_{2}$ & 1.9 & 0.8 & 0.4 & 0.5 \\
$\mathrm{Rc}$ & 0.5 & 0.2 & 0.1 & 0.1 \\
$\mathrm{Rd}$ & 0.4 & 0.2 & 0.1 & 0.2 \\
$\mathrm{Re}$ & 0.2 & 0.1 & 0.0 & 0.0 \\
$\mathrm{Rf}$ & 0.7 & 0.3 & 0.1 & 0.1 \\
$\mathrm{Rg}$ & 0.2 & 0.1 & 0.0 & 0.0 \\
\hline Total ginsenosides & 0.6 & 0.3 & 0.1 & 0.1 \\
\hline Diol/Triol & $\mathrm{b})$ & $\mathbf{2 . 0}$ & $\mathbf{1 . 0}$ & $\mathbf{1 . 2}$ \\
\hline
\end{tabular}

\footnotetext{
${ }^{\text {a) }}$ Sum of individual ginsenosides content, ${ }^{\text {b) }}$ Ginsenoside $\mathrm{Rb}_{1}+\mathrm{Rb}_{2}+\mathrm{Rc}+\mathrm{Rd} / \mathrm{Re}+\mathrm{Rf}+\mathrm{Rg}_{1}$
} 
Table 10. Seasonal ginsenoside average content of 10 years old Korean wild simulated ginseng (11 areas)

\begin{tabular}{ccccc}
\hline \hline Ginsenosides & \multicolumn{4}{c}{ Seasonal 10 years old Korean wild simulated ginseng (11 regions) } \\
\hline $\mathrm{Rb}_{1}$ & spring (May) & summer (July) & autumn (September) & winter (November) \\
$\mathrm{Rb}_{2}$ & 1.7 & 0.8 & 0.3 & 0.7 \\
$\mathrm{Rc}$ & 0.5 & 0.2 & 0.1 & 0.1 \\
$\mathrm{Rd}$ & 0.4 & 0.2 & 0.1 & 0.2 \\
$\mathrm{Re}$ & 0.2 & 0.1 & 0.0 & 0.1 \\
$\mathrm{Rf}$ & 0.5 & 0.2 & 0.1 & 0.2 \\
$\mathrm{Rg}$ & 0.2 & 0.0 & 0.0 & 0.0 \\
\hline Total ginsenosides $^{\mathrm{a})}$ & 0.5 & 0.2 & 0.1 & 0.2 \\
\hline Diol/Triol $^{\text {b) }}$ & $\mathbf{3 . 9}$ & $\mathbf{1 . 8}$ & $\mathbf{0 . 6}$ & $\mathbf{1 . 6}$ \\
\hline
\end{tabular}

${ }^{\text {a) }}$ Sum of individual ginsenosides content, ${ }^{\text {b) }}$ Ginsenoside $\mathrm{Rb}_{1}+\mathrm{Rb}_{2}+\mathrm{Rc}+\mathrm{Rd} / \mathrm{Re}+\mathrm{Rf}+\mathrm{Rg}_{1}$

spring (2.3) and autumn (2.2). Significance of the difference among the 10 years old groups of each places $(P<0.05)$.

In this study, HPLC analysis data for 3 times for each sample has been processed for average after the wild simulated ginseng samples of 11 representative cultivation places in South Korea. There were collected per number of years, season and region, which is considered as the data allowing verification of the current identity (representativeness) for ginseng saponin composition of the wild simulated ginseng of South Korea. Since 7 years old wild simulated ginseng consistently shows a higher content than 10 years old wild simulated ginseng for all season. It was considered that there is correlation between the number of years and the ginseng saponin composition. Since the wild simulated ginseng collected in spring showed the highest total saponin content. Followed by wild simulated ginseng collected in summer and winter with the wild simulated ginseng collected in autumn exhibiting a tendency for showing the lowest total saponin content, it is suggested that use of the wild simulated ginseng collected in spring should be considered for the raw material ginseng of the wild simulated ginseng with reinforced functionality.

Moreover, Tanaka et al. $^{23}$ reported that the cultivated ginseng collected in May has higher saponin content than the ginseng collected in November, and the wild simulated ginseng also showed the similar results in this study. Therefore, we conclude that the 7 years old Korean wild simulated ginseng especially collected in May is the most appropriate to use as the raw material for functionally improved healthcare products.

\section{Acknowledgements}

This study is supported by a grant (Project No.
S111415L020100) of the 'Forestry Technology Projects' provided by the Korea Forest Service.

\section{References}

(1) Ko, S. K.; Leem, K. H. Kor. J. Herbology 2009, 24, 169-172.

(2) Ko, S. K.; Im, B. O. Korean ginseng science; Yakupsinmusa: Seoul, 2009, p 33.

(3) Namba, T. The Encyclopedia of Wakan-Yaku with Color Pictures (I); Hoikusha: Osaka, 1980, p 1.

(4) Park, J. D. Korean J. Ginseng Sci. 1996, 20, 389-415.

(5) Sanada, S.; Kondo, N.; Shoji, J.; Tanaka, O.; Shibata, S. Chem. Pharm. Bull. 1974, 22, 421-428.

(6) Kitagawa, I.; Taniyama, T.; Shibuya, H.; Noda, T.; Yoshikawa, M. Yakugaku Zasshi 1987, 107, 495-505.

(7) Shibata, S.; Tanaka, O.; Ando, T.; Sado, M.; Tsushima, S.; Ohasawa, T. Chem. Pharm. Bull. 1966, 14, 595-600.

(8) Tanaka, O.; Nagai, M.; Shibata, S. Chem. Pharm. Bull. 1966, 14, 1150-1156.

(9) Lim, W.; Mudge, K. W.; Weston, L. A. Planta Med. 2007, 73, 7176.

(10) Lui, J. H. C.; Staba, E. J. J. Nat. Prod. 1980, 43, 340-346.

(11) Lim, W.; Mudge, K. W.; Vermeylen, F. J. Agric. Food Chem. 2005, 53, 8498-8505

(12) Kim, S. J.; Shin, S. S.; Seo, B. I.; Jee, S. Y. Kor. J. Herbology 2004, 19, 41-50.

(13) Hong, M. H.; Lim, H. K.; Park, J.; Jun, N. J.; Lee, Y. J.; Cho, M.; Cho, S. K. J. Korean Soc. Appl. Biol. Chem. 2008, 51, 102-107.

(14) Kim, J. H.; Kim, J. K. J. Korean Soc. Food Sci. Nutr. 2006, 35, 1315-1321.

(15) Kwon, K. R.; Cho, A. L.; Lee, S. G. J. Kor. Inst. Herb. Acupunc. 2003, 6, 7-27.

(16) Yun, S. N.; Moon, S. J.; Ko, S. K.; Im, B. O.; Chung, S. H. Arch. Pharm. Res. 2004, 27, 790-796.

(17) Lee, C. R.; Whang, W. K.; Shin, C. G.; Lee, H. S.; Han, S. T.; Im, B. O.; Ko, S. K. Korean J. Food Sci. Technol. 2004, 36, 847-850.

(18) Jang, J. G.; Lee, K. S.; Kwon, D. W.; Nam, K. Y.; Choi, J. H. Korean J. Food Nutr. 1983, 12, 37-40.

(19) Han, S. T.; Shin, C. G.; Yang, B. W.; Hahm, Y. T.; Sohn, U. D.; Im, B. O.; Cho, S. H.; Lee, B. Y.; Ko, S. K. Food Sci. Biotechnol. 2007, 16, 281-284.

(20) Lee, J. B.; Kim, M. U.; Cho, S. H.; Ko, S. K. Kor. J. Pharmacogn. 2017, 48, 255-259. 
(21) Lee, J. B.; Yang, B. W.; Park, J. D.; Ko, S. K. Yakhak Hoeji 2017, $61,274-280$.

(22) Ko, S. K.; Lee, K. H.; Hong, J. K.; Kang, S. A.; Sohn, U. D.; Im, B. O.; Han, S. T.; Yang, B. W.; Chung, S. H.; Lee, B. Y. Food Sci. Biotechnol. 2005, 14, 509-513.

(23) Kim, S. K.; Sakamoto, I.; Morimoto, K.; Sakata, M.; Yamasaki, K. K.; Tanaka, O. Planta Med. 1981, 42, 181-186.
Received July 17, 2018

Revised August 26, 2018 Accepted September 3, 2018 